\title{
Optimización de los Costos de Operación del Proceso de Electro-oxidación para una Planta de Tratamiento de Aguas Mediante Análisis Estadístico de Superficie de Respuesta
}

\author{
Edison GilPavas ${ }^{(1,2)}$, José Medina(1), Izabela Dobrosz-Gómez ${ }^{(1,2 b)}$, Miguel-Ángel Gómez $^{(1,2 a)}$ \\ (1) GIPAB: Grupo de Investigación en Procesos Ambientales, Departamento de Ingeniería de Procesos. \\ Universidad EAFIT, Cr 49 \#7 sur 50, Medellín-Colombia. (e-mail: egil@eafit.edu.co; jmedin11@eafit.edu.co) \\ (2)PRISMA: Grupo de Investigación en Procesos Reactivos Intensificados con Separación y Materiales \\ Avanzados. a Depto. de Ingeniería Química, Facultad de Ingeniería y Arquitectura. b Depto. de Física y \\ Química, Facultad de Ciencias Exactas y Naturales. Universidad Nacional de Colombia, Sede Manizales, \\ Campus La Nubia, Apartado Aéreo 127, Manizales, Caldas, Colombia \\ (e-mail: idobrosz-gomez@unal.edu.co; magomez@unal.edu.co)
}

Recibido Oct. 26, 2015; Aceptado Ene. 19, 2016; Versión final Feb. 7, 2016, Publicado Ago. 2016

\begin{abstract}
Resumen
Se efectuó la optimización estadística del costo de implementación y operación del tratamiento por electrooxidación de aguas residuales contaminadas con el colorante Amarillo 23. El objeto fue encontrar los valores de operación para la densidad de corriente, conductividad, y área de electrodos por unidad de volumen que minimicen el valor presente neto (VPN) de la operación mientras se mantiene una calidad de tratamiento establecida. Para alcanzar este objetivo se empleó la Metodología de Superficie de Respuesta acoplada a un diseño de experimentos Box-Behnken. Las condiciones óptimas encontradas fueron: Relación volumen tratado por área de electrodos: $9.076 \mathrm{~mL} / \mathrm{cm}^{2}$, conductividad: $4000 \mu \mathrm{S} / \mathrm{cm}$ y densidad de corriente $20 \mathrm{~mA} / \mathrm{cm}^{2}$, para las cuales el VPN predicho a 10 años es de 998636 USD y corresponde a un costo de $0.67 \mathrm{USD} / \mathrm{m}^{3}$ de agua tratada.
\end{abstract}

Palabras clave: tratamiento de aguas residuales; optimización; electroquímica; análisis de superficie de respuesta

\section{Optimization of the Operating Cost for the Electrochemical- Oxidation Process in a Water Treatment Plant Using Response Surface Statistical Analysis}

\begin{abstract}
The statistical optimization of the implementation and operational costs of an electrochemical-oxidation process for treatment of wastewater containing dye Yellow 23 was done. The aim was to optimize the operational parameters for the current density, conductivity, and area of electrodes per unit of volume in order to minimize the net present value (NPV) of the operation while maintaining a defined quality for the treated wastewater. To achieve this, the response surface methodology coupled to the Box-Behnken statistical design was used. The optimal conditions found were: a relationship of treated wastewater volume per area of electrodes of $9.076 \mathrm{~mL} / \mathrm{cm}^{2}$, conductivity $4000 \mu \mathrm{S} / \mathrm{cm}$, and current density $20 \mathrm{~mA} / \mathrm{cm}^{2}$. At optimal conditions, the NPV for a 10 year operation is 998636 USD, which corresponds to a cost of $0.68 \mathrm{USD} / \mathrm{m}^{3}$ of treated water.
\end{abstract}

Keywords: electrochemical; response surface methodology; optimization; wastewater 


\section{INTRODUCCIÓN}

La protección del medio ambiente y en especial de los recursos hídricos se ha convertido en un tema de gran interés público y social, por lo que las legislaciones en el mundo se hacen cada vez más estrictas en cuanto a los límites permisibles en las descargas de contaminantes a los cuerpos de agua (Panizza et al., 2007). Entre las diversas fuentes de polución, las aguas residuales que contienen colorantes sintéticos son un caso de especial atención debido a la gran cantidad con que son producidas y a las limitaciones de su tratamiento usando los métodos convencionales. La producción estimada de colorantes en el 2008 fue de $7 \times 10^{5}$ toneladas, de los cuales se estima que el $10 \%$ no se fija al producto final y se queda en el agua residual (Martínez y Brillas, 2009), los colorantes más usados son los tipo azo, que representan el $70 \%$ de la producción global y son ampliamente utilizados en la industria textil y de alimentos (Ramírez et al., 2013). Los procesos convencionales de coagulación-floculación, filtración, adsorción, y biodegradación no son eficientes para su tratamiento, por lo cual es necesario el desarrollo e implementación de tratamientos con tecnologías emergentes para alcanzar los nuevos y futuros estándares de calidad del agua.

Los Procesos Avanzados de Oxidación (PAO's) han sido materia de estudio en las últimas dos décadas como tecnologías alternativas para el tratamiento de aguas con diversos tipos de contaminantes orgánicos. En efecto, se han publicado gran cantidad de estudios en los que se demuestra su eficiencia en el tratamiento de aguas residuales con colorantes, herbicidas, residuos de refinerías y otros contaminantes (Martínez y Brillas, 2009, Moreira et al., 2015, Palma-Goyes et al., 2014, GilPavas et al., 2014b, Ramírez et al., 2013). Entre los PAO's, la electro-oxidación (EO) es un proceso que ha demostrado ser eficiente en el tratamiento de aguas residuales industriales. Comninellis (1994) propuso el mecanismo de la EO, donde la reacción inicial corresponde a la hidrólisis del agua para la formación de radicales hidroxilo $\left({ }^{\circ} \mathrm{OH}\right)$ adsorbidos en la superficie del ánodo de acuerdo a la ecuación (1):

$\mathrm{M}+\mathrm{H}_{2} \mathrm{O} \rightarrow \mathrm{M}(\cdot \mathrm{OH})+\mathrm{H}^{+}+\mathrm{e}^{-}$

Donde M denota la superficie del electrodo. La superficie del electrodo interactúa de forma débil con el ${ }^{\circ} \mathrm{OH}$ generado, permitiéndole oxidar directamente la materia orgánica presente en la solución. La reacción (2) describe el proceso de oxidación para alcanzar la mineralización completa de los contaminantes, donde los productos principales son el $\mathrm{CO}_{2}$ y agua (Martínez y Brillas, 2009).

$\mathrm{M}(\cdot \mathrm{OH})+\mathrm{R} \rightarrow \mathrm{M}+\mathrm{CO}_{2}+\mathrm{H}_{2} \mathrm{O}+\mathrm{H}^{+}+\mathrm{e}-$

La EO ha sido implementada recientemente a escala piloto, y se han publicado varios estudios acerca de su escalado. Sin embargo, la mayoría de ellos se han centrado en la cuantificación del efecto de condiciones de operación como densidad de corriente (i), área de electrodos (Ae), concentración de electrolito, concentración del contaminante (Ci), pH, y tiempo de reacción (Anglada y Urtiaga, 2009).El objetivo ha sido maximizar la degradación de contaminantes en el agua, sin tener en cuenta la minimización de los costos de operación (Martínez y Brillas., 2009; Anglada y Urtiaga, 2010; Yavuzet al., 2010, Ramírez et al., 2013, GilPavas et al., 2014a, GilPavas et al., 2015), lo cual es fundamental para la implementación de esta tecnología a escala industrial.Ramírez et al.(2013) evaluaron el proceso EO para la remoción del colorante azo naranja de metilo a escala piloto con un caudal de $12 \mathrm{~L} / \mathrm{min}$ y usando la MSR, los autores encontraron que las condiciones óptimas para la degradación fueron pH:3 y un área de electrodos de $64 \mathrm{~cm}^{2}$, obteniendo una degradación del $94 \%$ en 138 minutos con una i de $31 \mathrm{~mA} / \mathrm{cm}^{2}$; no obstante, no se considera que es posible alcanzar la misma degradación con otras condiciones que reduzcan el costo total de la operación, como se demuestra en la presente investigación. Anglada y Urtiaga, 2010, evaluaron el proceso EO a escala piloto, para aguas residuales provenientes de los lixiviados de relleno sanitario, obteniendo una degradación completa de la DQO, lo que demuestra la viabilidad de este proceso para tratar aguas altamente contaminadas provenientes de diversas industrias, sin embargo, los autores, no utilizaron un modelo estadístico de optimización y no hay claridad en cómo los parámetros de operación tienen incidencia sobre los costos del proceso.

El presente estudio propone un modelo de optimización que minimiza el valor presente neto (VPN) en la implementación y operación del proceso de EO una planta de tratamiento de aguas residuales. Esto con el fin de definir las condiciones de operación que minimizan los costos del proceso mientras se mantiene la calidad de tratamiento establecida. En esta investigación se evaluó el proceso de degradación del contaminante modelo Amarillo 23 (A23), colorante tipo azo, ampliamente utilizado en la industria farmacéutica y de alimentos. Se empleó la metodología de superficie de respuesta (MSR) acoplada a un diseño de experimentos Box-Behnken (DBB), que permite reducir el número total de experimentos, en comparación con los diseños factoriales completos, lo que implica menor consumo de reactivos y horas de trabajo de laboratorio. Además, permite desarrollar un modelo matemático para establecer la significancia 
estadística del efecto de los factores evaluados, así como la interacción entre factores. Así, se evaluó el efecto de la densidad de corriente (i), el volumen de reacción por área de electrodo (V/A), y la conductividad $(\mathrm{Cd})$ en el tiempo requerido para que cada una de las condiciones experimentales alcance una degradación decolorante del $90 \%$.Se fijó como variable de respuesta el VPN de una operación a 10 años para una planta que procesa $400 \mathrm{~m}^{3} /$ día de agua residual.

\section{MATERIALES Y EQUIPOS}

\section{Reactivos y equipos}

Como contaminante modelo se utilizó el colorante $\mathrm{A} 23\left(\mathrm{C}_{16} \mathrm{H}_{9} \mathrm{~N}_{4} \mathrm{Na}_{3} \mathrm{O}_{9} \mathrm{~S}_{2} \geq 89 \% \mathrm{w} / \mathrm{w}\right.$, Colorquímica $\left.{ }^{\mathrm{TM}}\right)$ usado sin purificación adicional. Los demás reactivos fueron suministrados por Merck y usados en el estado que se recibieron. Las soluciones fueron preparadas con agua de un sistema mili-Q. El pH fue ajustado con soluciones de $\mathrm{H}_{2} \mathrm{SO}_{4}$ al $99.1 \%$ de pureza y la conductividad fue ajustada con $\mathrm{NaCl}$ anhídrido. La mezcla de reacción se agitó a $240 \mathrm{rpm}$ y el voltaje fue regulado con una fuente BK-Precision (0-30V, 0-5 A). Las muestras resultantes de cada uno de los ensayos fueron analizadas usando un espectrofotómetro UVVisSpectronics Genesys 2PC, longitud de onda de $424 \mathrm{~nm}$, correspondiente a la máxima absorbancia del colorante A23. El pH y la conductividad de cada una de las muestras fueron determinadas mediante el equipo multiparamétrico ( $\mathrm{pHmetro} \mathrm{y} \mathrm{coductímetro)} \mathrm{Thermo} \mathrm{Scientific} \mathrm{Orion} 5$ stars.

\section{Sistema de reacción}

Los ensayos fueron realizados en un reactor de acrílico continuamente agitado de $80 \mathrm{~mL}$ de capacidad, con dos electrodos ubicados en forma vertical: ánodo rectangular de grafito y cátodo rectangular de titanio con 1 $\mathrm{cm}$ de separación entre ellos. Las dimensiones de los electrodos varían de acuerdo al diseño de experimentos. El montaje para el proceso EO se muestra en la Fig.1. A la solución de agua contaminada con $\mathrm{A} 23$, se le adicionó $\mathrm{NaCl}$ hasta alcanzar la conductividad requerida según lo planteado en el diseño de experimentos; posteriormente, esta mezcla se alimentó a la celda electrolítica y se hizo pasar una corriente eléctrica por los electrodos para obtener el agua tratada.

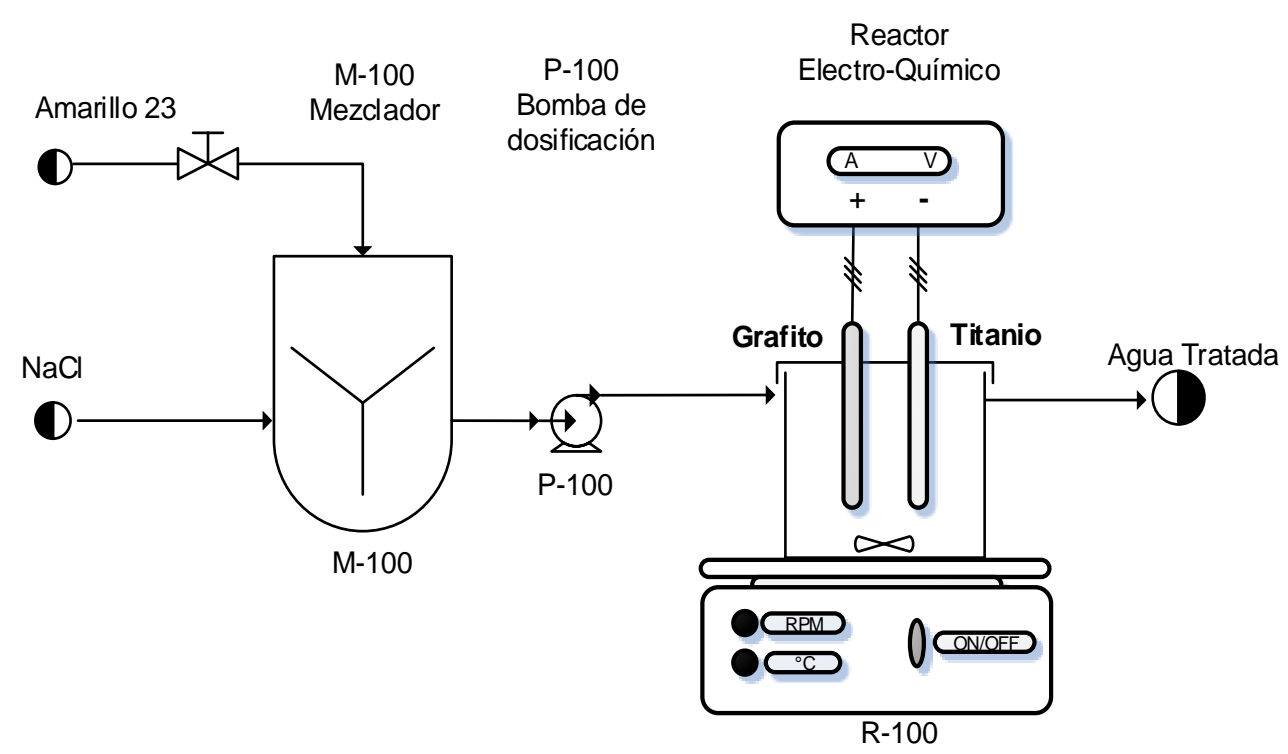

Fig.1: Sistema de reacción

\section{Diseño estadístico de experimentos}

El objetivo del diseño estadístico de experimentos es encontrar las condiciones de operación que minimizan los costos, mientras se mantiene la calidad del tratamiento, la cual se establece cuando se obtenga una reducción del $90 \%$ del color y se determina el tiempo que le toma a las diferentes condiciones de operación en alcanzar esta condición. Con el fin de lograr este objetivo, se empleó la MSR para evaluar y determinar las condiciones óptimas de operación que minimizan los costos de operación, considerando todos los efectos y las interacciones de las variables presentadas en la Tabla 1. Los factores evaluados fueron: la relación entre el volumen tratado y el área de los electrodos (V/A), conductividad (Cd), y densidad de corriente (i). 
Tabla 1: Factores o Variables evaluados con sus respectivos niveles

\begin{tabular}{|l|c|c|c|}
\hline \multicolumn{4}{|c|}{ Factores Codificados } \\
\hline & -1 & 0 & 1 \\
\hline Variable & Nivel 1 & Nivel 2 & Nivel 3 \\
\hline V/A $\left(\mathrm{mL} / \mathrm{cm}^{2}\right)$ & 4,6 & 9,8 & 15 \\
\hline $\mathrm{Cd}(\mu \mathrm{S} / \mathrm{cm})$ & 1000 & 2500 & 4000 \\
\hline $\mathrm{i}\left(\mathrm{mA} / \mathrm{cm}^{2}\right)$ & 10 & 15 & 20 \\
\hline
\end{tabular}

La MSR se desarrolló acoplado al DBB, método especial para analizar procesos con varios factores cuya principal ventaja es que son rotables y reducen el número de pruebas experimentales para realizar la optimización (GilPavas et al., 2014a). La programación de los experimentos y análisis estadístico de los datos se realizó con ayuda del software estadístico Statgraphics Centurion XVI. El DBB consta de 15 experimentos, que fueron efectuados de manera aleatoria y con réplica con el fin de evitar algún sesgo sistemático. Este modelo evalúa cada una de las variables a 3 niveles y los resultados obtenidos se ajustan a un polinomio de regresión de segundo orden, ecuación (3), donde $\beta_{0}, \beta_{i}, \beta_{i i}, \beta_{i j}$ son los coeficientes de regresión para el intercepto, el término lineal, cuadrático, y de sus interacciones, respectivamente, y $X_{i}$ y $X_{j}$ son las variables independientes (Montgomery, 2005).

$Y_{i}=\beta_{0}+\sum_{1}^{3} \beta_{i} x_{i}+\sum_{1}^{3} \beta_{i i} x_{i i}^{2}+\sum_{1}^{3} \sum_{1}^{3} \beta_{i j} x_{i} x_{j}$

La variable de respuesta principal es el VPN, con el propósito de implementar una planta de tratamiento con las condiciones óptimas de operación determinadas, que se calculan de acuerdo al modelo descrito en la siguiente sección.

\section{Modelo de cálculo para el valor presente neto (VPN)}

La variable respuesta, VPN, se calculó a 10 años de la puesta en marcha y funcionamiento de una planta que opera con cada una de las condiciones experimentales y un flujo de $400 \mathrm{~m}^{3} / \mathrm{dí}$; para lo cual, en cada ensayo se debe medir el tiempo que toma la reacción en alcanzar la disminución de color establecida (90\%), y se utiliza como base para su dimensionamiento. El cálculo VPN considera el costo de la puesta en marcha del reactor más el costo de operación del proceso EO. Las ecuaciones (4-5) muestran el modelo de cálculo del VPN a 10 años con un interés del $1 \%$ efectivo anual.

$V P N 10$ años $(U S D)=\left[\sum_{i=1}^{10} \frac{\text { Costo Operación }}{(1-0.01)^{i}}+\right.$ Costo Reactor $]$

Costo Anual Operación $\left(\frac{U S D}{a \tilde{n} o}\right)=$ Costo $\mathrm{NaCl}+$ Costo Electricidad + Costo Electrodos

Para calcular el costo del rector es necesario dimensionar el volumen de reacción, que depende del tiempo de reacción, hallado experimentalmente para alcanzar una reducción del colorante del $90 \%$ y corresponde al tamaño del batch calculado mediante la ec. (6). Una vez definido el tamaño del reactor,se procede a estimar su costo mediante la metodología propuesta por Turton (Turton et al., 2014).

Tamaño Batch $\left(m^{3}\right)=\frac{\text { Volumen tratado poraño }}{\frac{\text { Horastrabajo año }}{\text { Tiempo } R x n}}$

El costo de operación se compone del costo la electricidad, del electrolito $(\mathrm{NaCl})$, y de los electrodos, calculados de acuerdo a las ecuaciones (7-9)

$$
\text { Costo Electricidad }(\$ / a \tilde{n} o)=\frac{K W h}{a \tilde{n} o} * \text { Costo } K W h
$$

Costo $\frac{N a C l}{a \tilde{\text { ño }}}(\$ / a \tilde{n} o)=\frac{\text { Volumen }}{a \tilde{n} o} *$ Concentración $\mathrm{NaCl} *$ US\$NaCl

Costo Electrodos $(\$ / a \tilde{n} o)=$ Costo $\frac{U S \$}{m^{2}} *$ Área Electrodo

Con el VPN a 10 años se procede con el análisis del diseño de experimentos. 


\section{RESULTADOS}

La programación de cada uno de los experimentos del DBB se presenta en la tabla 2.Además, en ella se observan los resultados obtenidos del tiempo requerido en alcanzar el $90 \%$ dela degradación del colorante y el VPN a 10 años de operación. Se observa que el ensayo que reporta el mejor VPN es el 3, con V/A: 9,8 $\mathrm{mL} / \mathrm{cm}^{2}, \mathrm{Cd}: 4000 \mu \mathrm{S} / \mathrm{cm}$ y i: $20 \mathrm{~mA} / \mathrm{cm}^{2}$. Con estas condiciones de operación es posible alcanzar la reducción de color en 4 minutos de operación. Se observa también que aunque otras condiciones de operación, como el ensayo 6 , requieren el mismo tiempo de reacción, su costo de operación es mayor.

Tabla 2: Resultados experimentales DBB

\begin{tabular}{|c|c|c|c|c|c|}
\hline Ensayo & $V / A\left(m L / \mathrm{cm}^{2}\right)$ & $C d(\mu S / \mathrm{cm})$ & $i\left(\mathrm{~mA} / \mathrm{cm}^{2}\right)$ & $\begin{array}{c}\text { Tiempo (min) (para una } \\
\text { degradación del 90\% de color) }\end{array}$ & VPN 10 años (USD) \\
\hline 1 & 9,8 & 2500 & 15 & 18,82 & $\$ 2 ’ 560.417,75$ \\
\hline 2 & 9,8 & 1000 & 20 & 36 & $\$ 66^{\prime} 457.809,15$ \\
\hline 3 & 9,8 & 4000 & 20 & 4 & $\$ 1 ' 640.164,65$ \\
\hline 4 & 15 & 1000 & 15 & 76 & $\$ 7 ’ 207.821,05$ \\
\hline 5 & 9,8 & 4000 & 10 & 15 & $\$ 2 ' 287.933,16$ \\
\hline 6 & 4,6 & 4000 & 15 & 4 & $\$ 1 ' 976.329,10$ \\
\hline 7 & 9,8 & 2500 & 15 & 18,5 & $\$ 2 ' 498.995,90$ \\
\hline 8 & 15 & 2500 & 20 & 26,3 & $\$ 2 ' 932.174,21$ \\
\hline 9 & 15 & 4000 & 15 & 20,5 & $\$ 2 ' 415.740,03$ \\
\hline 10 & 9,8 & 2500 & 15 & 18 & $\$ 2{ }^{\prime} 481.703,90$ \\
\hline 11 & 4,6 & 2500 & 20 & 4,8 & $\$ 2 ' 150.749,67$ \\
\hline 12 & 4,6 & 1000 & 15 & 18,5 & $\$ 5 ' 475.294,27$ \\
\hline 13 & 4,6 & 2500 & 10 & 14,3 & $\$ 3 ’ 002.909,54$ \\
\hline 14 & 9,8 & 1000 & 10 & 40 & $\$ 4^{\prime} 081.108,11$ \\
\hline 15 & 15 & 2500 & 10 & 59,2 & $\$ 3718.476,56$ \\
\hline
\end{tabular}

Los coeficientes de regresión y los p-valor encontrados con el análisis de la varianza, ANOVA, se presentan en la tabla 3. El p-valor provee información del grado de compatibilidad de los resultados con la hipótesis nula y verifica la significancia estadística de cada factor. Con un intervalo de confianza del $95 \%$ los $p$-valor inferiores a 0,05 son estadísticamente significativos. De acuerdo a los resultados obtenidos, el efecto de la conductividad y su término cuadrático son los dos que presentaron p-valor menores a 0.05 , por tanto, son estadísticamente significativos. Además, la interacción $\mathrm{Cd}$-i (BC) presenta un p-valor cercano a 0,05, por lo que se considera relevante para la evaluación de los resultados.

Tabla 3: Tabla ANOVA DBB

\begin{tabular}{|c|c|c|}
\hline Coeficiente & Estimado & $p$-valor \\
\hline Constante & $-4,35 \mathrm{E}+06$ & - \\
\hline $\mathrm{A}: \mathrm{VA}$ & 212520 & 0,0849 \\
\hline $\mathrm{B}: \mathrm{Cd}$ & $\underline{2013,16}$ & $\underline{0,0003}$ \\
\hline $\mathrm{C}: \mathrm{i}$ & -376898 & 0,9599 \\
\hline $\mathrm{AA}$ & $-20144,5$ & 0,1442 \\
\hline $\mathrm{AB}$ & $-41,446$ & 0,3341 \\
\hline $\mathrm{AC}$ & $-633,245$ & 0,9587 \\
\hline $\mathrm{BB}$ & $\underline{-0,53794}$ & $\underline{0,0121}$ \\
\hline $\mathrm{BC}$ & $\underline{100,816}$ & $\underline{0,0505}$ \\
\hline CC & 4293,41 & 0,7471 \\
\hline $\mathrm{R}^{2}=95,46 \%$ & & \\
\hline $\mathrm{R}^{2}$ Adj (ajustada mediante grados de libertad) & $=87,30 \%$ \\
\hline \multicolumn{3}{|c|}{}
\end{tabular}

En la Figura 2 se muestra el diagrama de Pareto, herramienta estadística para resaltar aquellos factores que exhiben un mayor efecto acumulativo sobre la variable respuesta y representa gráficamente los efectos estandarizados para cada factor, desde el más alto hasta el más bajo. Aquellos factores que son estadísticamente significativos son los que sobrepasan la línea vertical y los signos positivo (+) y negativo (-) 
indican si al modificar un nivel de un factor este tiene un efecto directamente o inversamente proporcional sobre la variable respuesta. Se evidencia que la $\mathrm{Cd}$ tiene el mayor efecto sobre la variable respuesta y que a mayor conductividad se obtendrá un menor VPN.

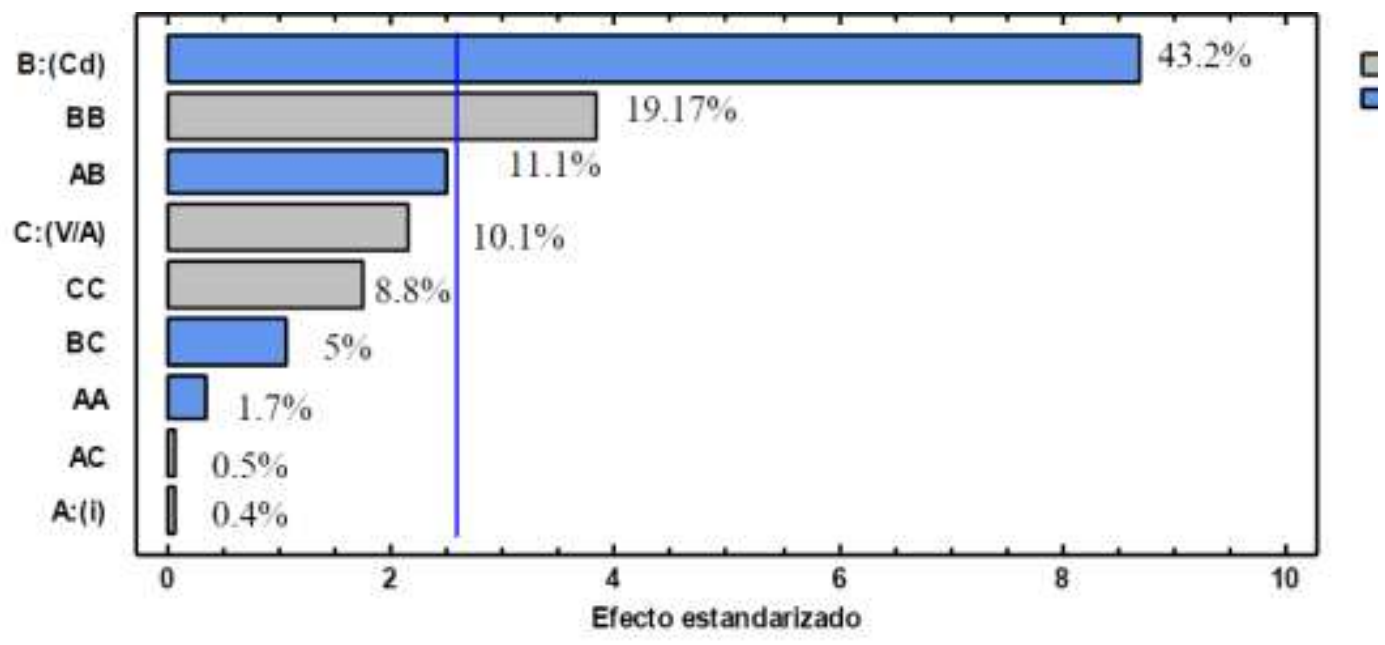

Fig.2: Diagrama de Pareto

En diversos estudios donde se ha evaluado el proceso EO empleando ánodo de grafito y $\mathrm{NaCl}$ como electrolito para la degradación de colorantes orgánicos, como Índigo, Rojo Congo y Naranja de Metilo se han encontrado que las remociones de colorante se incrementan con la densidad de corriente, área de electrodo y concentración de electrolito (Cameselle et al., 2005, Wang, 2003).Sin embargo, los resultados de la tabla 3 indican que sólo la última variable tiene un efecto significativo sobre el VPN como variable respuesta. Por tal motivo, para una correcta interpretación de los resultados, se realiza un análisis por separado del tiempo requerido para alcanzar la degradación del colorante establecido y de cada uno de los elementos que intervienen en el costo de operación (costo de electrodos, electrolito, y energía eléctrica).En la tabla 4 se presentan los $p$-valor y los efectos estimados para cada una de estas variables.

Tabla 4: Efectos y p-valor para el tiempo de degradación del colorante y elementos del costo

\begin{tabular}{|c|c|c|c|c|c|c|c|c|}
\hline \multirow{3}{*}{ Factores } & \multicolumn{2}{|c|}{ Tiempo Rxn requerido } & \multicolumn{2}{|c|}{ \$Energía Eléctrica } & \multicolumn{2}{|c|}{ \$ Electrodos } & \multicolumn{2}{|c|}{ \$ Electrolito (NaCl) } \\
\cline { 2 - 9 } & $\begin{array}{c}\text { Efectos } \\
\text { Estimados }\end{array}$ & $p$-valor & $\begin{array}{c}\text { Efectos } \\
\text { Estimados }\end{array}$ & $p$-valor & $\begin{array}{c}\text { Efectos } \\
\text { Estimados }\end{array}$ & $p$-valor & $\begin{array}{c}\text { Efectos } \\
\text { Estimados }\end{array}$ & $p$-valor \\
\hline A:VA & 35,1 & $\underline{0,0002}$ & 47776,5 & 0,1537 & 27411,5 & 0,2669 & 0 & 1 \\
\hline B:Cd & $-31,75$ & $\underline{0,0003}$ & -320133 & $\underline{0,0001}$ & -159528 & $\underline{0,0008}$ & 95470,5 & $\underline{0}$ \\
\hline C:i & $-14,35$ & $\underline{0,0094}$ & 79414,9 & $\underline{0,0383}$ & $-84397,5$ & $\underline{0,012}$ & 0 & 1 \\
\hline AA & 13,71 & 0,0448 & 61815,2 & 0,1996 & 61318,5 & 0,1161 & 0 & 1 \\
\hline AB & $-20,5$ & $\underline{0,009}$ & $-49737,7$ & 0,2709 & -13797 & 0,6752 & 0 & 1 \\
\hline AC & $-11,7$ & 0,0645 & 9247,5 & 0,8272 & 19768,4 & 0,5521 & 0 & 1 \\
\hline BB & 8,91 & 0,1443 & -177738 & $\underline{0,0042}$ & 34336,8 & 0,3364 & $-0,01$ & $\underline{0}$ \\
\hline BC & $-3,5$ & 0,5111 & -140150 & $\underline{0,0175}$ & $-17989,3$ & 0,5872 & 0 & 1 \\
\hline CC & 1,71 & 0,7534 & $-31673,4$ & 0,4832 & 20247,8 & 0,5582 & 0 & 1 \\
\hline
\end{tabular}

En la tabla 4 se observa que, a diferencia del VPN, se obtienen p-valores inferiores a 0.05 para los diferentes elementos del costo y tiempo de reacción requerido. El signo (+) o (-) en el efecto estimado, indica si al incrementar este factor se tiene un efecto directamente (+) o inversamente proporcional (-) sobre la variable respuesta. Es decir, para la densidad de corriente (i) se encontró un efecto estimado de $-14,35$ sobre el tiempo de reacción, por lo que se interpreta que se obtienen menores tiempos de reacción con el incremento de i. Los resultados muestran que la intensidad de corriente además de tener efecto significativo sobre el tiempo de reacción requerido, también lo tiene sobre el costo de energía eléctrica y sobre el costo de los electrodos; no obstante, en la tabla 3 se muestra que no tenía efecto significativo sobre el VPN. Esto se puede explicar debido a que un incremento de (i) reduce el tiempo de reacción requerido, por lo que se necesitaría un reactor y electrodos más pequeños lo que disminuiría los costos del proceso. Sin embargo, el aumento en la (i) incrementa los costos relacionados con la energía eléctrica y su efecto global sobre los costos no es significativo. 
La conductividad fue la variable que resultó más significativa sobre el VPN. En la Tabla 4 se observa que esta disminuye el tiempo de reacción, reduce el costo en energía eléctrica y el costo de los electrodos, mientras que incrementa el costo en el consumo de electrolito. Sin embargo, este incremento en el costo de reactivos es mucho menor que los beneficios en la reducción de los otros elementos del costo y consecuentemente resulta mucho mejor trabajar con alta conductividad. Este resultado concuerda con diversos estudios en los que se ha encontrado que mayores concentraciones de $\mathrm{NaCl}$ reducen los tiempos de degradación de colorantes (Wang, 2003, Sanroman et al., 2004, Panizza et al., 2007), fenómeno que se explica debido a que la conductividad mejora el flujo de la corriente eléctrica a través de la solución, requiriendo un voltaje mucho menor para alcanzar una determinada intensidad de corriente y por lo tanto, se reduce el consumo de la energía. Además, el $\mathrm{NaCl}$ promueve la formación de otras especies oxidantes como $\mathrm{Cl}_{2}$ and $\mathrm{ClO}^{-}$, que atacan los colorantes y ayudan a incrementar la eficiencia del proceso (Golder et al., 2005; Cameselle et al. 2005).

En la Figura 3 se presenta el diagrama de interacciones entre la Cd-i sobre el VPN. Se observa que para la $\mathrm{Cd}$ de $4000 \mu \mathrm{S} / \mathrm{cm}$ siempre se encuentran menores costos que para $1000 \mu \mathrm{S} / \mathrm{cm}$. Por lo tanto, esta es la condición más favorable para la operación. En cuanto a la (i), el menor costo se obtiene cuando se trabaja con $20 \mathrm{~mA} / \mathrm{cm}^{2} \mathrm{y}$ la mayor $\mathrm{Cd}$. En este caso, un incremento en la (i) resulta favorable para disminuir los costos, cuando se trabaja a la máxima $\mathrm{Cd}$, debido a que a niveles bajos de $\mathrm{Cd}$ existe una mayor resistencia al paso de la electricidad.

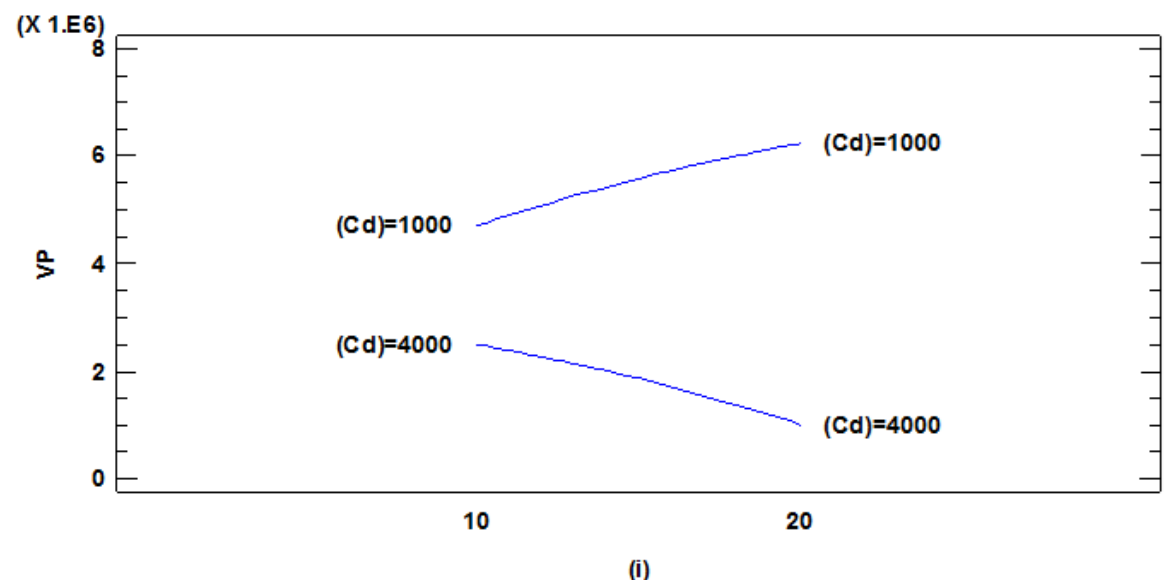

Fig.3: Diagrama de interacciones entre Cd-i sobre el VPN

En la Figura 4 se presenta el diagrama de interacciones entre la Cd-V/A. Se observa que los menores costos se encuentran con las menores relaciones V/A; es decir, cuando se emplea una mayor área de electrodos por volumen de agua tratada. Sin embargo, cuando se emplea una Cd de $4000 \mu \mathrm{S} / \mathrm{cm}$, la diferencia en el VPN no es significativa con el cambio en el área de electrodos. El análisis de optimización encontró que las condiciones que maximizan la variable respuesta son: V/A: $9,076 \mathrm{~mL} / \mathrm{cm}^{2}, \mathrm{Cd}: 4000 \mu \mathrm{S} / \mathrm{cm}$ y (i) $20 \mathrm{~mA} / \mathrm{cm}^{2}$, para las cuales el VPN predicho a 10 años es de 998,636 USD, lo que corresponde a un costo de $0,68 \mathrm{USD} / \mathrm{m}^{3}$ de agua tratada.

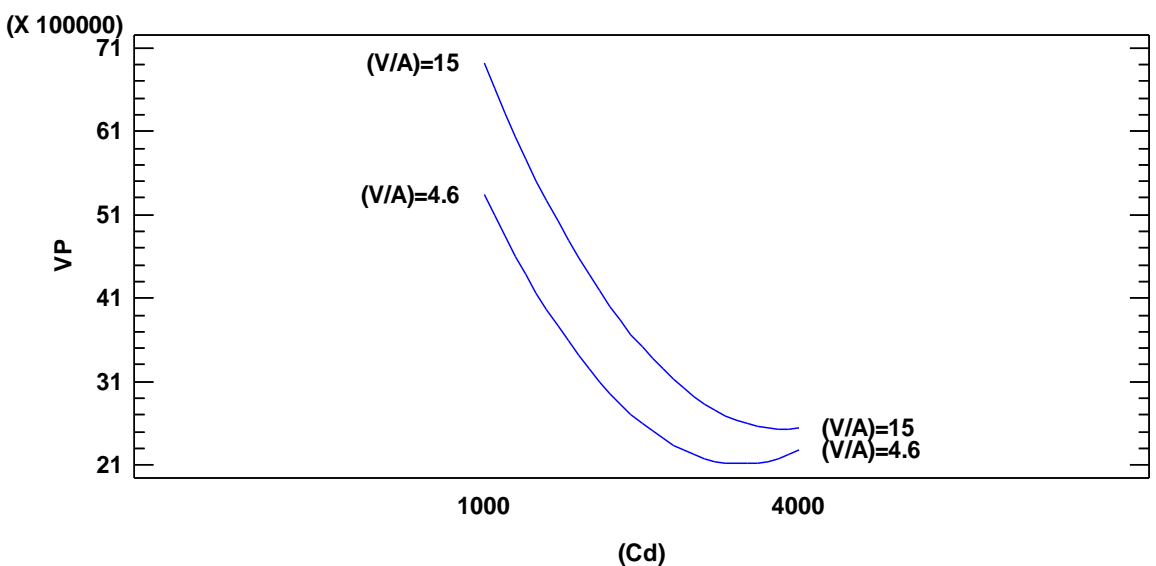

Fig.4: Diagrama de interacciones entre $\mathrm{Cd}-\mathrm{V} / \mathrm{A}$ sobre el VPN 
La Figura 5 presenta el diagrama de superficie de respuesta, en la que se observa claramente como el VPN disminuye a medida que se incrementa la $\mathrm{Cd}$ de la solución, mientras que para la relación V/A el costo mínimo se encuentra cerca a los $9 \mathrm{~mL} / \mathrm{cm}^{2}$. Los resultados muestran que es posible llegar a tratamientos de la misma calidad variando las condiciones de operación. Sin embargo, las diferentes condiciones incurrirán en diferentes costos.

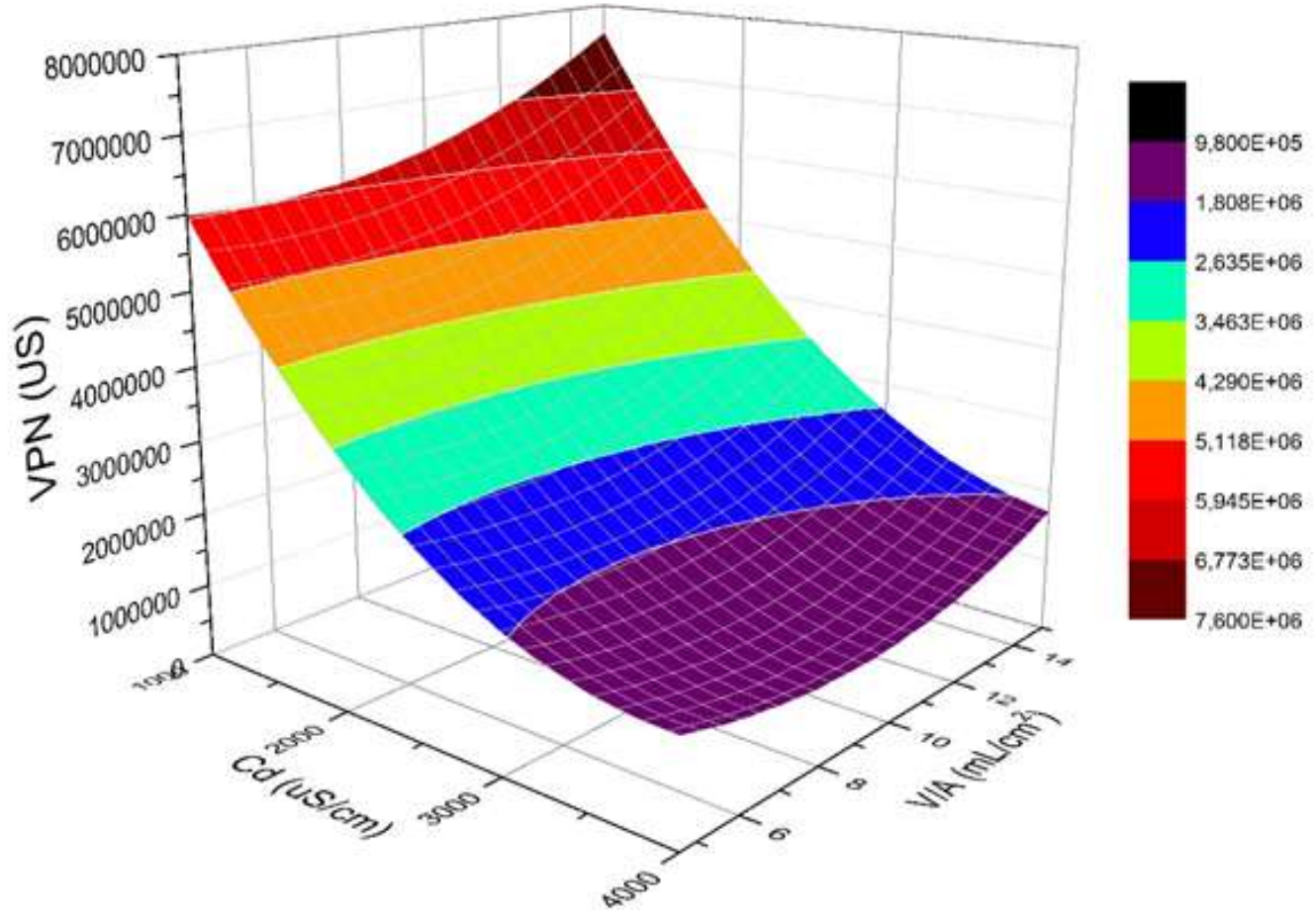

Fig.5: Diagrama de superficie de respuesta

Los resultados manifiestan que el proceso EO es un tratamiento eficaz para la degradación de colorante en aguas residuales textiles, alcanzando remociones superiores al $90 \%$ en tiempos de operación muy cortos, entre 4 y 20 minutos. En el trabajo se destaca que el modelo de optimización se aplicó sobre costo del proceso, incluyendo el costo de los equipos, consumo de reactivos y de energía y no únicamente sobre la eficiencia en la degradación de colorante, como lo reportan en la gran mayoría de investigaciones (Anglada y Urtiaga, 2010; Ramírez et al., 2013).El modelo desarrollado resulta de gran utilidad para llevar estos procesos a escala industrial, donde el costo es la principal limitante para su implementación.

\section{CONCLUSIONES}

EL proceso EO con ánodo de grafito se puede aplicar de manera eficiente para la degradación del colorante azo A23. Las siguientes conclusiones se pueden extraer de los resultados de la investigación: (i) Con el análisis de optimización se determinó que las condiciones que maximizan la variable respuesta son: V/A: $9,076 \mathrm{~mL} / \mathrm{cm}^{2}$, Cd: $4000 \mu \mathrm{S} / \mathrm{cm}$ y i: $20 \mathrm{~mA} / \mathrm{cm}^{2}$, obteniendo un VPN predicho a 10 años de 998,636 USD, que corresponde a un costo de $0,68 \mathrm{USD} / \mathrm{m}^{3} \mathrm{de}$ agua tratada. (ii) Los factores estadísticamente más significativos para el proceso al evaluar su impacto en el VPN son la $\mathrm{Cd}$, su término cuadrático y la interacción Cd-i. La Cd es también significativa sobre el tiempo de reacción, obteniéndose menores tiempos a mayores valores de Cd, lo que permitiría la construcción de un reactor más pequeño y de menor costo de electrodos.

Adicionalmente, al ser mayor la Cd se reduce el voltaje requerido para alcanzar la i y por ende, el costo de la electricidad disminuye. Estos efectos implican que, a pesar del costo adicional que ocasiona la incorporación del $\mathrm{NaCl}$, el aumento de la $\mathrm{Cd}$ es beneficioso para el costo total. (iii) Todos los factores individuales son estadísticamente significativos sobre el tiempo requerido para alcanzar la degradación de color establecida, logrando menores tiempos al incrementar la (i), el área del electrodo, y la concentración de electrolito. El factor V/A tiene el mayor efecto sobre el tiempo requerido para la degradación del colorante, donde la velocidad de reacción es mayor mientras menor sea la relación V/A.(iv)Debido a que en todos los tratamientos se alcanza la misma calidad del agua residual este modelo de optimización de costos con DBB resultó muy útil para buscar las condiciones más rentables y acercar estos procesos a su escalado industrial. 


\section{AGRADECIMIENTOS}

Este trabajo se ha desarrollado con el apoyo de la Dirección de investigación de la Universidad EAFIT y del El Departamento Administrativo de Ciencias, Tecnología e Innovación COLCIENCIAS, programa Jóvenes Investigadores.

\section{REFERENCIAS}

Anglada, A. Urtiaga, I. Ortiz., Pilot scale performance of the electro-oxidation of landfill leachate at borondoped diamond anodes, doi: 10.1021/es802748c, Environ. Sci. Technol. 43(6), 2035-2040 (2009)

Anglada, A. M. Urtiaga, I. Ortiz., Laboratory and pilot plant scale study on the electrochemical oxidation of landfill leachate, doi:10.1016/j.jhazmat.2010.05.073, Journal of Hazardous Materials 181, 729-735 (2010)

Cameselle, M. Pazos, M.A. Sanromán.,Selection of an electrolyte to enhance the electrochemical decolourisation of indigo, doi:10.1016/j.chemosphere.2005.01.018, Optimization and scale-up, Chemosphere 60(8), 1080-1086 (2005)

Comninellis, Ch., Electrocatalysis in the electrochemical conversion/combustion of organic pollutants for water treatments, doi:10.1016/0013-4686(94)85175-1, Electrochim. Acta 39(11-12), 1857-1862 (1994)

GilPavas E. y otros tres autores, Decolorization and mineralization of Yellow 5 (E102) by UV/Fe ${ }^{2}+/ \mathrm{H}_{2} \mathrm{O}_{2}$ process, doi:org/10.1016/j.crci.2015.08.001. Optimization of the operational conditions by Response Surface Methodology. Comptes Rendus Chimie, 18(10), 1152-1160 (2015)

GilPavas E. y otros tres autores, Statistical optimization of industrial textile wastewater treatment by electrochemical methods, doi: 10.1007/s10800-014-0767-y,J. of Applied Electroch., 44, 1421-1430 (2014a)

GilPavas, E. Dobrosz-Gómez, I. Gómez-García, M, Degradación y Mineralización de Tartrazina mediante Electro-oxidación: Optimización de las Condiciones de Operación, doi: 10.4067/S071807642014000400018, Inf. tecnol., 25(4), 163-174 (2014b)

Golder A.K. y otros tres autores, Electrocoagulation of methylene blue and eosin yellowish using mild steel electrodes, doi:10.1016/j.jhazmat.2005.06.032, J. Hazard. Mater., B127(1-3), 134-140 (2005)

Martínez C.A, E. Brillas, Decontamination of wastewaters containing synthetic organic dyes by electrochemical methods: A general review, doi:10.1016/j.apcatb.2008.09.017, Applied Catalysis B: Environmental, 87(3-4), 105-145 (2009)

Moreira F. C. y otros seis autores, Incorporation of electrochemical advanced oxidation processes in a multistage treatment system for sanitary landfill leachate, doi:10.1016/j.watres.2015.05.036, Water Research 81, 375-387 (2015)

Montgomery Douglas C., Design and Analysis of Experiments, John Wiley \& Sons, Inc. Estados Unidos de América. INC, ISBN: 0471316490 (2005)

Palma-Goyes R. y otros tres autores, Comparative degradation of indigo carmine by electrochemical oxidation and advanced oxidation processes, doi:10.1016/j.electacta.2014.06.096, Electroc. Acta, 140, 427433(2014)

Panizza M. y otros tres autores, Electrochemical degradation of methylene blue,doi:10.1016/j.seppur.2006.10.010, Sep. Purif. Technol. 54 (3), 382-387 (2007)

Ramírez $\mathrm{C}$ y otros 7 autores, Electrochemical oxidation of methyl orange azo dye at pilot flow plant using BDD technology, doi:10.1016/j.jiec.2012.09.010,J. of Industrial and Engineering Chemistry 19(2), 571-579 (2013)

Sanroman, M. Pazos, C. Cameselle, Optimization of electrochemical decolorization process of an azo dye, methyl orange, doi: 10.1002/jctb.1098, J. Chem. Technol. Biotechnol. 79(12), 1349-1353(2004)

Turton, R.C y otros tres autores, Analysis, Synthesis and Design of Chemical Processes, Fourth Edition, Prentice Hall, (2012)

Wang, C.T. Decolorization of Red Congo with three-dimensional flow-by packed bed electrodes,doi:10.1007/s11356-011-0532-2, Environ. Sci. Health A, 38, 399-413(2004)

Yavuz, A.S Koparal, U.B. Öğütveren. Treatment of petroleum refinery wastewater by electrochemical methods,doi:10.1016/j.desal.2010.03.013, Desalination, 258(1-3), 201-205 (2010) 